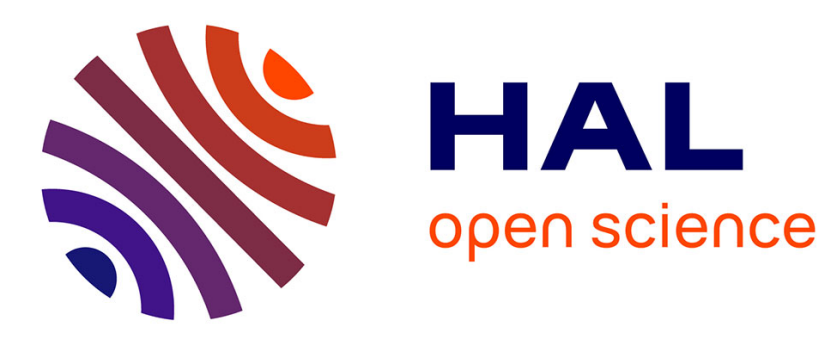

\title{
Constitutive Equation Gap
}

\author{
Stéphane Pagano, Marc Bonnet
}

\section{To cite this version:}

Stéphane Pagano, Marc Bonnet. Constitutive Equation Gap. Full-Field Measurements and Identification in Solid Mechanics, John Wiley \& Sons, 26p., 2012, 978-1-84821-294-7. hal-00827980

\section{HAL Id: hal-00827980 \\ https://hal.science/hal-00827980}

Submitted on 30 May 2013

HAL is a multi-disciplinary open access archive for the deposit and dissemination of scientific research documents, whether they are published or not. The documents may come from teaching and research institutions in France or abroad, or from public or private research centers.
L'archive ouverte pluridisciplinaire HAL, est destinée au dépôt et à la diffusion de documents scientifiques de niveau recherche, publiés ou non, émanant des établissements d'enseignement et de recherche français ou étrangers, des laboratoires publics ou privés. 


\section{Chapitre 10}

\section{Constitutive equation gap}

\subsection{Introduction}

In this chapter we examine the concept of constitutive equation gap (CEG) as a tool for the identification of parameters associated with behavior models for solid materials. The concept of CEG is based, in its simplest form (small strain hypothesis, equilibrium, linear elastic constitutive behavior) on cost-functions of the form

$$
E(\boldsymbol{v}, \boldsymbol{\tau}, \mathcal{B})=\frac{1}{2} \int_{\Omega}(\boldsymbol{\tau}-\mathcal{B}: \varepsilon[\boldsymbol{v}]): \mathcal{B}^{-1}:(\boldsymbol{\tau}-\mathcal{B}: \varepsilon[\boldsymbol{v}]) \mathrm{d} V
$$

(where $\boldsymbol{v}$ is a displacement field, $\boldsymbol{\tau}$ a stress field, and $\mathcal{B}$ a possibly-heterogeneous elasticity tensor), expressing a quadratic gap in the verification of the constitutive law. Weighting by the tensor $\mathcal{B}$ confers units of energy to the CEG.

The concept of CEG was initially proposed for error estimation in the finite element method [LAD 83]. It then turned out to be also a powerful tool for identification, especially with many applications in model updating. Essentially equivalent concepts have been proposed in other contexts for solving inversion problems, such as the electrostatic energy functionals of Kohn and Vogelius [KOH 84, KOH 90]. Two important characteristics of CEG functionals are (i) their strong and clear physical meaning, and (ii) their additive character with respect to the structure, allowing the definition of local error indicators over substructures.

As will be discussed in this chapter for different situations, the minimization of a constitutive equation gap is in principle applicable to any identification problem for

Chapitre rédigé par Stéphane PAGAno, Laboratoire de Mécanique et Génie Civil, UMR CNRS 5508, Université Montpellier 2, 34095 Montpellier et Marc Bonnet, POems, UMR 7231 CNRS-INRIA-ENSTA, 91120 Palaiseau. 
which overdetermined data are available. In particular, the latter need not necessarily consist of field measurements. In fact, many applications of the CEG concern structural model updating from vibrational data (see Section 10.5). The CEG method is nonetheless well suited to the exploitation of field measurements.

Initially, we will focus on the identification of heterogeneous linear elastic properties under static (i.e. equilibrium) conditions and within the small-strain assumption (Section 10.2). An extension of this formulation to the identification of heterogeneous elastoplastic constitutive parameters is then described (section 10.3). The algorithms presented in these two sections exploit kinematic field measurements in a plane, and assume either plane-strain or plane-stress conditions. The remainder of the chapter will be devoted to a more succinct discussion of the possibilities offered by the construction of ERC functionals based on Legendre-Fenchel error density (Section 10.4), and a synthetic presentation of CEG formulations suitable for structural dynamics and vibrations (section 10.5).

\subsection{CEG in the linear elastic case : heterogeneous behavior and full-field mea-} surement

The equilibrium of a generic elastic solid $\Omega$ of boundary $\partial \Omega$ is governed by three sets of equations, namely the equilibrium equations

$$
\left\{\begin{aligned}
\operatorname{div} \boldsymbol{\sigma} & =0 \quad \text { in } \Omega, \\
\boldsymbol{\sigma} . \boldsymbol{n} & =\overline{\boldsymbol{T}} \quad \text { on } S_{f},
\end{aligned}\right.
$$

the kinematic compatibility conditions

$$
\left\{\begin{aligned}
\varepsilon[\boldsymbol{u}] & =\frac{1}{2}\left(\nabla \boldsymbol{u}+\nabla^{t} \boldsymbol{u}\right) \quad \text { in } \Omega, \\
\boldsymbol{u} & =\overline{\boldsymbol{u}} \quad \text { on } S_{u}
\end{aligned}\right.
$$

and the constitutive equation

$$
\boldsymbol{\sigma}=\mathcal{A}(\boldsymbol{x}): \varepsilon[\boldsymbol{u}] .
$$

where $\boldsymbol{u}$ is the in-plane displacement, $\varepsilon[\boldsymbol{u}]$ the linearized strain tensor associated with $\boldsymbol{u}, \boldsymbol{\sigma}$ the Cauchy stress tensor, and $\boldsymbol{n}$ the outward unit normal on $\partial \Omega$. Quantities $\overline{\boldsymbol{u}}$ and $\overline{\boldsymbol{T}}$ appearing in the boundary conditions (10.1) and (10.2) denote prescribed displacements and tractions, respectively. Surfaces $S_{u}$ and $S_{f}$ are such that $S_{u} \cup S_{f}=\partial \Omega$ and $S_{u} \cap S_{f}=\emptyset$, so as to define well-posed boundary conditions. The elasticity tensor $\mathcal{A}$ may be constant (homogeneous material) or space-dependent (heterogeneous material). If the elastic properties are isotropic, they are described in terms of two independent moduli, such as Lamé constants $\lambda$, $\mu$, or Young's modulus $E$ and Poisson's ratio $\nu$. 
The usual problem, often referred to as direct, consists of computing fields $\boldsymbol{u}, \boldsymbol{\sigma}$ knowing the geometry $\Omega$ of the solid, the elasticity tensor $\mathcal{A}$ and well-posed boundary data $\overline{\boldsymbol{u}}, \overline{\boldsymbol{T}}$. Exact solutions are limited to very specific classes of geometries and boundary conditions ; the forward problem is usually solved numerically, most often using the finite element method (FEM).

The identification of constitutive parameters is another type of problem, referred to as inverse. Incomplete knowledge of the tensor-valued function $\mathcal{A}(\boldsymbol{x})$, or of moduli used in the definition of $\mathcal{A}$, must be offset by overdetermined data. In other words, the boundary data $\overline{\boldsymbol{u}}, \overline{\boldsymbol{T}}$ appearing in (10.1) and (10.2) must be supplemented by experimental information. Such additional data can take various forms.

In this chapter the focus is on situations where field measurements are available either in $\Omega$ or on $\partial \Omega$. The three most frequently encountered situations are then :

1) Measurements $\hat{\boldsymbol{u}}$ of the displacement field $\boldsymbol{u}$ and complete well-posed boundary data $\overline{\boldsymbol{u}}, \overline{\boldsymbol{T}}$ are available. The strain tensor components are then obtained by differentiating the displacement field and are therefore regarded as known.

2) Measurements $\hat{\boldsymbol{u}}$ of the displacement field $\boldsymbol{u}$ and boundary data $\overline{\boldsymbol{u}}$ are available, together with only incomplete static boundary data; e.g. only resultant forces associated with $\overline{\boldsymbol{T}}$ are known.

3) Overdetermined boundary data are available (for example, $\overline{\boldsymbol{T}}$ known on $S_{f}$ and $\overline{\boldsymbol{u}}$ known on $\partial \Omega$ ), with displacement and strain fields inside the solid remaining to be determined.

This chapter focuses on situations of type 1) or 2) above. Case 3) is touched upon in Section 10.5, and also corresponds to a data structure suitable for the reciprocity gap method [AND 92, AND 96] (see Chapter 13).

The constitutive equation gap (CEG) measures the distance between a stress field $\tau$ and another stress field resulting from the application of a constitutive model to a displacement field $\boldsymbol{v}$. The CEG between $\boldsymbol{\tau}$ and $\boldsymbol{v}$, under the assumption of linear elastic behavior characterized by the (possibly heterogeneous) elasticity tensor $\mathcal{B}$, is defined by

$$
E(\boldsymbol{v}, \boldsymbol{\tau}, \mathcal{B})=\frac{1}{2} \int_{\Omega}(\boldsymbol{\tau}-\mathcal{B}: \varepsilon[\boldsymbol{v}]): \mathcal{B}^{-1}:(\boldsymbol{\tau}-\mathcal{B}: \varepsilon[\boldsymbol{v}]) \mathrm{d} V
$$

The presence of the compliance tensor $\mathcal{B}^{-1}$ in the integral confers units of energy to $E(\boldsymbol{v}, \boldsymbol{\tau}, \mathcal{B})$. It can actually be shown that

$$
E(\boldsymbol{v}, \boldsymbol{\tau}, \mathcal{B})=\mathcal{P}(\boldsymbol{v}, \mathcal{B})+\mathcal{P}^{\star}(\boldsymbol{\tau}, \mathcal{B}),
$$

with $\mathcal{P}$ and $\mathcal{P}^{\star}$ respectively denoting the potential energy and complementary energy.

The usefulness of the concept of CEG is emphasized by the following remarks : 
1) For a well-posed boundary value problem such as that defined by equations (10.1), (10.2), (10.3) ( $\overline{\boldsymbol{u}}, \overline{\boldsymbol{T}}, \mathcal{A}$ known, $\boldsymbol{u}, \varepsilon, \boldsymbol{\sigma}$ unknown), spaces $\mathcal{C}$ of kinematically admissible displacement fields and $\mathcal{S}$ of statically admissible stress fields are defined by :

$$
\begin{aligned}
\mathcal{C}\left(\overline{\boldsymbol{u}}, S_{u}\right) & =\left\{\boldsymbol{v} \mid v_{i} \in \mathrm{H}^{1}(\Omega), \boldsymbol{v}=\overline{\boldsymbol{u}} \text { on } S_{u}\right\}, \\
\mathcal{S}\left(\overline{\boldsymbol{T}}, S_{f}\right) & =\left\{\boldsymbol{\tau} \in \mathrm{H}_{\mathrm{div}}(\Omega), \operatorname{div} \boldsymbol{\tau}=0 \text { in } \Omega, \text { and } \boldsymbol{\tau} \cdot \boldsymbol{n}=\overline{\boldsymbol{T}} \text { on } S_{f}\right\},
\end{aligned}
$$

where the space $\mathrm{H}_{\text {div }}(\Omega)$ of tensor-valued fields is defined by

$$
\mathrm{H}_{\mathrm{div}}(\Omega)=\left\{\boldsymbol{\tau} \mid \tau_{i j}=\tau_{j i}, \tau_{i j} \in \mathrm{L}^{2}(\Omega), \tau_{i j, j} \in \mathrm{L}^{2}(\Omega)\right\} .
$$

The solution $(\boldsymbol{u}, \boldsymbol{\sigma})$ of the boundary value problem is then characterized by

$$
(\boldsymbol{u}, \boldsymbol{\sigma})=\underset{(\boldsymbol{v}, \boldsymbol{\tau}) \in \mathcal{C} \times \mathcal{S}}{\arg \min } E(\boldsymbol{v}, \boldsymbol{\tau}, \mathcal{A}) \quad \text { et } \quad E(\boldsymbol{u}, \boldsymbol{\sigma}, \mathcal{A})=0 .
$$

2) For a constitutive parameter identification problem for which (for instance) the elasticity tensor $\mathcal{A}$ is unknown, the definitions (10.6a) and (10.6b) of spaces of admissible fields can be modified to include all available experimental information on the displacements and stresses. The elasticity tensor $\mathcal{A}$ can then be identified by minimizing the constitutive equation gap :

$$
\mathcal{A}=\underset{\mathcal{B}}{\arg \min } \mathcal{E}(\boldsymbol{B}) \quad \text { with } \quad \mathcal{E}(\mathcal{B})=\min _{(\boldsymbol{v}, \boldsymbol{\tau}) \in \mathcal{C} \times \mathcal{S}} E(\boldsymbol{v}, \boldsymbol{\tau}, \mathcal{B})
$$

This minimization problem is the essence of the constitutive equation gap method (CEGM). It consists of an alternating directions method for which a partial minimization with respect to $(\boldsymbol{v}, \boldsymbol{\tau})$ is followed by a partial minimization with respect to $\mathcal{B}$.

Two variants of the CEGM will now be described. Both require the availability of a measurement $\overline{\boldsymbol{u}} \in\left(\mathrm{H}^{1}(\Omega)\right)^{2}, \Omega \subset \mathbb{R}^{2}$ of the displacement field $\boldsymbol{u}$.

\subsubsection{First variant : exact enforcement of kinematic measurements}

In this variant, the measured displacement field $\overline{\boldsymbol{u}}$ is introduced directly into the CEG functional. In addition, the values $\boldsymbol{g}_{i}$ of linear observation functionals $L_{i}$ of the traction vector $\boldsymbol{\tau} . \boldsymbol{n}$ (where $\boldsymbol{n}$ is the outward normal vector) are assumed to be known :

$$
L_{1}(\boldsymbol{\tau} . \boldsymbol{n})=\boldsymbol{g}_{1} \operatorname{sur} \Gamma_{1}, \ldots, L_{N}(\boldsymbol{\tau} . \boldsymbol{n})=\boldsymbol{g}_{N} \operatorname{sur} \Gamma_{N},
$$

where $\Gamma_{i} \subset \partial \Omega$. In order to exploit experimental data, two instances of such functionals occur commonly : 
(i) $L(\boldsymbol{\tau} . \boldsymbol{n}) \equiv \boldsymbol{\tau} . \boldsymbol{n}=\boldsymbol{g}$ (in particular $\boldsymbol{g}=\mathbf{0}$ for the stress-free part of the boundary),

(ii) $L(\boldsymbol{\tau} . \boldsymbol{n}) \equiv \int_{\Gamma} \boldsymbol{\tau} . \boldsymbol{n} d l=\boldsymbol{R}$ ( $\boldsymbol{R}$ is the known resultant load associated with a distributed loading over a part $\Gamma$ of the boundary).

All this leads us to seek $\mathcal{A}$ as a solution to the minimization problem

$$
\mathcal{A}=\arg \min _{\mathcal{B} \in \mathcal{A}} \mathcal{E}(\boldsymbol{B}) \quad \text { with } \quad \mathcal{E}(\boldsymbol{B})=\min _{\boldsymbol{\tau} \in \mathcal{S}} E(\overline{\boldsymbol{u}}, \boldsymbol{\tau}, \mathcal{B}) .
$$

In this case, the space of admissible stress fields is defined as

$$
\begin{aligned}
\mathcal{S}\left(\overline{\boldsymbol{T}}, S_{f}\right)=\left\{\boldsymbol{\tau} \in \mathrm{H}_{\mathrm{div}}(\Omega), \operatorname{div} \boldsymbol{\tau}=0 \text { in } \Omega,\right. & \\
& \left.L_{1}(\boldsymbol{\tau} . \boldsymbol{n})=\boldsymbol{g}_{1}, \ldots, L_{N}(\boldsymbol{\tau} . \boldsymbol{n})=\boldsymbol{g}_{N}\right\}
\end{aligned}
$$

instead of (10.6b). Each functional $L_{i}:\left(\mathrm{H}^{-1 / 2}\left(\Gamma_{i}\right)\right)^{2} \rightarrow \boldsymbol{E}_{i}$ is linear and continuous (where $\boldsymbol{E}_{i}$ in a Banach space). $\mathcal{S}$ is assumed to be nonempty (from an abstract point of view, an open question is to find suitable conditions on the data $\boldsymbol{g}_{1}, \ldots, \boldsymbol{g}_{N}$ in such a way that this assumption be satisfied).

Two possible simple choices may be considered for the space of admissible elasticity (stiffness or compliance) tensors $\mathcal{A}$

$$
\begin{array}{r}
\mathcal{A}_{1}=\left\{\boldsymbol{B} \in \mathbb{R}^{3 \times 3} ; B_{i j}=B_{j i}, \boldsymbol{B} \boldsymbol{x}: \boldsymbol{x} \geq \alpha|\boldsymbol{x}|^{2}, \alpha>0, \forall \boldsymbol{x},\right. \\
\boldsymbol{B} \boldsymbol{x}: \boldsymbol{y} \leq M|\boldsymbol{x}||\boldsymbol{y}|, M>0, \forall \boldsymbol{x}, \boldsymbol{y}\}, \\
\mathcal{A}_{2}=\left\{B_{i j} \text { piecewise linear } ; B_{i j}=B_{j i}, \boldsymbol{B} \boldsymbol{x}: \boldsymbol{x} \geq \alpha|\boldsymbol{x}|^{2}, \alpha>0, \forall \boldsymbol{x},\right. \\
\boldsymbol{B} \boldsymbol{x}: \boldsymbol{y} \leq M|\boldsymbol{x}||\boldsymbol{y}|, M>0, \forall \boldsymbol{x}, \boldsymbol{y}\} .
\end{array}
$$

The most general choice would be

$$
\begin{array}{r}
\mathcal{A}_{3}=\left\{\boldsymbol{B} \in\left(\mathrm{L}^{\infty}(\Omega)\right)^{3 \times 3} ; B_{i j}=B_{j i}, \boldsymbol{B} \boldsymbol{x}: \boldsymbol{x} \geq \alpha|\boldsymbol{x}|^{2}, \alpha>0, \forall \boldsymbol{x},\right. \\
\boldsymbol{B} \boldsymbol{x}: \boldsymbol{y} \leq M|\boldsymbol{x}||\boldsymbol{y}|, M>0, \forall \boldsymbol{x}, \boldsymbol{y}\} .
\end{array}
$$

With these notations, the following relations can be established for the CEG functional $E(\overline{\boldsymbol{u}}, . .):. \mathcal{S} \times \mathcal{A} \rightarrow \mathbb{R}$

Proposition 1 The following properties for the CEG functional hold true

(a) $E(\overline{\boldsymbol{u}}, \boldsymbol{\tau}, \mathcal{B}) \geq 0, \forall(\boldsymbol{\tau}, \mathcal{B}) \in \mathcal{S} \times \mathcal{A}$,

(b) $E\left(\overline{\boldsymbol{u}}, \boldsymbol{\tau}^{\star}, \mathcal{B}^{\star}\right)=0$ if and only if (10.3) is true with $\boldsymbol{\sigma}=\boldsymbol{\tau}^{\star}$ and $\mathcal{A}=\mathcal{B}^{\star}$.

(c) Functional $E(\overline{\boldsymbol{u}}, . .$, .) is convex on $\mathcal{S} \times \mathcal{A}$. 
Proof Properties (a) and (b) are obvious, since the elasticity tensor $\mathcal{B}$ belongs to space $\mathcal{A}$. The convexity of $E(\overline{\boldsymbol{u}}, . .$.$) remains to be established. To this end, the CEG func-$ tional is first rewritten as

$$
E(\overline{\boldsymbol{u}}, \boldsymbol{\tau}, \mathcal{B})=\int_{\Omega}\left\{\frac{1}{2} \varepsilon[\overline{\boldsymbol{u}}]: \mathcal{B}: \varepsilon[\overline{\boldsymbol{u}}]+\frac{1}{2} \boldsymbol{\tau}: \mathcal{B}^{-1}: \boldsymbol{\tau}-\boldsymbol{\tau}: \varepsilon[\overline{\boldsymbol{u}}]\right\} \mathrm{d} V
$$

and the convexity of function $\psi(\boldsymbol{\tau}, \mathcal{B}):=\frac{1}{2} \boldsymbol{\tau}: \mathcal{B}^{-1}: \boldsymbol{\tau}$ on $\mathcal{S} \times \mathcal{A}$ is investigated. A first-order Taylor expansion yields

$$
\psi(\boldsymbol{\tau}, \mathcal{B})=\psi\left(\boldsymbol{\tau}_{0}, \mathcal{B}_{0}\right)+\psi^{\prime}\left(\boldsymbol{\tau}_{0}, \mathcal{B}_{0} ; \boldsymbol{\tau}-\boldsymbol{\tau}_{0}, \mathcal{B}-\boldsymbol{B}_{0}\right)+\psi\left(\boldsymbol{\tau}-\mathcal{B}: \boldsymbol{B}_{0}^{-1}: \boldsymbol{\tau}_{0}, \mathcal{B}\right),
$$

where $\psi^{\prime}\left(\boldsymbol{\tau}_{0}, \mathcal{B}_{0} ; \boldsymbol{\tau}, \mathcal{B}\right)=-\frac{1}{2} \boldsymbol{\tau}_{0}:\left(\mathcal{B}_{0}^{-1}: \mathcal{B}: \mathcal{B}_{0}^{-1}\right): \boldsymbol{\tau}_{0}+\boldsymbol{\tau}: \mathcal{B}_{0}^{-1}: \boldsymbol{\tau}_{0}$. The desired convexity property follows from the non-negativity of $\psi$ by virtue of the definition of the set $\mathcal{A}$.

Algorithm 1 (Alternate direction method) The functional $E(\overline{\boldsymbol{u}}, . .,$.$) is minimized on$ $\mathcal{S} \times \mathcal{A}$ using an alternate direction method:

- Initialization of the algorithm with $\left(\sigma^{0}, \mathcal{A}^{0}\right)$

- For $\left(\boldsymbol{\sigma}^{n}, \mathcal{A}^{n}\right)$ known, find $\boldsymbol{\sigma}^{n+1}$ and $\mathcal{A}^{n+1}$ by successively solving

step 1: $E\left(\overline{\boldsymbol{u}}, \boldsymbol{\sigma}^{n+1}, \mathcal{A}^{n}\right) \leq E\left(\overline{\boldsymbol{u}}, \boldsymbol{\tau}, \mathcal{A}^{n}\right), \quad \forall \boldsymbol{\tau} \in \mathcal{S}$

step 2: $E\left(\overline{\boldsymbol{u}}, \boldsymbol{\sigma}^{n+1}, \mathcal{A}^{n+1}\right) \leq E\left(\overline{\boldsymbol{u}}, \boldsymbol{\sigma}^{n+1}, \mathcal{B}\right), \quad \forall \mathcal{B} \in \mathcal{A}$

- Convergence test.

In Step 1, the stress solution $\boldsymbol{\sigma}^{n+1}$ is constrained by the equilibrium equations (10.1) and the linear observations (10.9). Several methods can be used for this purpose, and three possible representations of $\boldsymbol{\sigma}^{n+1}$ will be presented next.

Partial minimization of $E\left(\bar{u}, ., \mathcal{A}^{n}\right)$ with respect to the admissible stress

First possibility : stress-based method using Q1 element. Minimization is performed by using a Q1 finite element interpolation of the admissible stress fields. For a planestress problem, the unknowns at step $n+1$ are $\left(\sigma_{x x}^{n+1}, \sigma_{y y}^{n+1}, \sigma_{x y}^{n+1}\right)$ at each node. Enforcing the equilibrium equations and resultant load measurements contributing to the definition of $\mathcal{S}$ via Lagrange multipliers leads to the solution of the variational problem

$$
\inf _{\boldsymbol{\tau} \in \mathrm{H}_{\mathrm{div}}(\Omega)} \sup _{\gamma, \lambda_{i}} E(\overline{\boldsymbol{u}}, \boldsymbol{\tau}, \mathcal{B})+\int_{\Omega} \gamma \cdot \operatorname{div} \boldsymbol{\tau} \mathrm{d} V+\sum_{i=1}^{N} \lambda_{i}\left[L_{i}(\boldsymbol{\tau} . \boldsymbol{n})-g_{i}\right] .
$$

The fact that Lagrange multipliers are involved leads to a substantial increase in the size of the governing linear system to be solved for the computation of the stresses, and a deterioration of its condition number. Instead, the computation of the stresses can be made more direct by resorting to an Airy stress function. 
Second possibility: Airy function. This formulation is naturally equilibrated [GER 86]. On each element of the mesh, the Airy function is assumed to be a third-degree polynomial with respect to each coordinate :

$$
\varphi(x, y)=\sum_{i=0}^{3} \sum_{j=0}^{3} a_{i j} x^{i} y^{j} .
$$

Consistent with this choice, the three stress components can be directly derived from this potential :

$$
\begin{aligned}
& \tau_{x x}(x, y)=\varphi_{, y y}=\sum_{i=0}^{3} \sum_{j=2}^{3} j(j-1) a_{i j} x^{i} y^{j-2} \\
& \tau_{y y}(x, y)=\varphi_{, x x}=\sum_{i=2}^{3} \sum_{j=0}^{3} i(i-1) a_{i j} x^{i-2} y^{j} \\
& \tau_{x y}(x, y)=-\varphi_{, x y}=-\sum_{i=1}^{3} \sum_{j=1}^{3} i j a_{i j} x^{i-1} y^{j-1}
\end{aligned}
$$

Whatever the choice of coefficients $a_{i j}$, $\operatorname{div} \boldsymbol{\tau}=0$. The variational problem is similar to (10.13) without the term enforcing local equilibrium. At each node, the unknowns, formulated in terms of the stress function, are $\left(\varphi, \varphi_{, x}, \varphi_{, y}, \varphi_{, x y}\right)$.

The present choice (10.14) of a bicubic potential enables a sufficiently rich representation of the stresses, since they are at least piecewise linear functions of each Cartesian coordinate. Note that this special interpolation method does not achieve inter-element stress continuity :

- the stress component $\sigma_{x x}^{n+1}$ is continuous only along the axis $O x$,

- the stress component $\sigma_{y y}^{n+1}$ is continuous only along the axis $O y$,

- the stress component $\sigma_{x y}^{n+1}$ is continuous.

Stress continuity can also be imposed, for example using Lagrange multipliers.

Third possibility : displacement-based representation. Stress fields are represented in the form $\boldsymbol{\sigma}^{n+1}=\mathcal{A}^{n}: \varepsilon[\boldsymbol{w}]$ in terms of displacement fields $\boldsymbol{w}$ (built using a finite element interpolation), and constraints on $\boldsymbol{\sigma}^{n+1}$ involved in the definition of $\mathcal{S}$ are enforced via a weak formulation associated with the finite element basis.

Partial minimization of $E\left(\bar{u}, \sigma^{n+1},.\right)$ over the admissible elasticity tensors

The minimization of $\mathcal{E}(\mathcal{B})$ is explicit. In the examples in this chapter, material properties are assumed to be constant in each element of the mesh. The capability of grouping elements into subsets $\omega_{i}$ with constant material properties has also been 
implemented. This possibility is particularly interesting when large subregions made of some specific material exist in $\Omega$ (as with e.g. coarse-grained polycrystalline steels, composite materials...). In such situations, the components of the elasticity tensor are given by very simple, explicit, formulae.

Two cases will be studied here, depending on whether we are considering (in the framework of plane elasticity) elastic behavior with cubic symmetry (three independent parameters) or isotropic symmetry (two independent parameters). The identification of parameters for cubic elasticity will be presented first, isotropic elasticity then being a special case. Similar explicit expressions are also available for threedimensional elasticity [CON 95].

Elasticity with cubic symmetry. For elastic behavior with cubic symmetry, in plane stress, the elasticity tensor $\mathcal{A}$ can be written in terms of three parameters as

$$
\mathcal{A}=\left[\begin{array}{ccc}
a_{1} & a_{2} & 0 \\
a_{2} & a_{1} & 0 \\
0 & 0 & a_{3}
\end{array}\right],
$$

with $a_{1}=E / 1-\nu^{2}, a_{2}=-\nu a_{1}, a_{3}=2 G$ and $E, \nu, G$ respectively denoting the Young's modulus, Poisson's ratio and shear modulus. The determination of $E, \nu, G$ is made simple by expressing tensors $\varepsilon[\bar{u}]$ and $\sigma$ in the principal directions of the elasticity tensor. The CEG functional then takes a simple form, and for each subdomain $\omega_{i}$ the entries of $\mathcal{A}$ (directly linked to $E^{i}, \nu^{i}, G^{i}$ ) are found to be given by

$$
\begin{aligned}
2 a_{1}^{i} & =\sqrt{\frac{\int_{\omega_{i}}\left(\tau_{x x}+\tau_{y y}\right)^{2} \mathrm{~d} V}{\int_{\omega_{i}}\left(\varepsilon_{x x}+\varepsilon_{y y}\right)^{2} \mathrm{~d} V}}+\sqrt{\frac{\int_{\omega_{i}}\left(\tau_{x x}-\tau_{y y}\right)^{2} \mathrm{~d} V}{\int_{\omega_{i}}\left(\varepsilon_{x x}-\varepsilon_{y y}\right)^{2} \mathrm{~d} V}}, \\
2 a_{2}^{i} & =\sqrt{\frac{\int_{\omega_{i}}\left(\tau_{x x}+\tau_{y y}\right)^{2} \mathrm{~d} V}{\int_{\omega_{i}}\left(\varepsilon_{x x}+\varepsilon_{y y}\right)^{2} \mathrm{~d} V}}-\sqrt{\frac{\int_{\omega_{i}}\left(\tau_{x x}-\tau_{y y}\right)^{2} \mathrm{~d} V}{\int_{\omega_{i}}\left(\varepsilon_{x x}-\varepsilon_{y y}\right)^{2} \mathrm{~d} V}}, \\
2 a_{3}^{i} & =\sqrt{\frac{\int_{\omega_{i}} \tau_{x y}^{2} \mathrm{~d} V}{\int_{\omega_{i}} \varepsilon_{x y}^{2} \mathrm{~d} V}} .
\end{aligned}
$$

Isotropic elasticity. For isotropic elastic behavior, parameters $E$ and $\nu$ can be determined using a technique identical to that previously developed, noting that now $a_{3}=a_{1}-a_{2}$. The two independent entries of $\mathcal{A}$ are obtained as

$$
\begin{aligned}
& 2 a_{1}^{i}=\sqrt{\frac{\int_{\omega_{i}}\left(\tau_{x x}+\tau_{y y}\right)^{2} \mathrm{~d} V}{\int_{\omega_{i}}\left(\varepsilon_{x x}+\varepsilon_{y y}\right)^{2} \mathrm{~d} V}}+\sqrt{\frac{\int_{\omega_{i}}\left(\tau_{x x}-\tau_{y y}\right)^{2}+\tau_{x y}^{2} \mathrm{~d} V}{\int_{\omega_{i}}\left(\varepsilon_{x x}-\varepsilon_{y y}\right)^{2}+\varepsilon_{x y}^{2} \mathrm{~d} V}}, \\
& 2 a_{2}^{i}=\sqrt{\frac{\int_{\omega_{i}}\left(\tau_{x x}+\tau_{y y}\right)^{2} \mathrm{~d} V}{\int_{\omega_{i}}\left(\varepsilon_{x x}+\varepsilon_{y y}\right)^{2} \mathrm{~d} V}}-\sqrt{\frac{\int_{\omega_{i}}\left(\tau_{x x}-\tau_{y y}\right)^{2}+\tau_{x y}^{2} \mathrm{~d} V}{\int_{\omega_{i}}\left(\varepsilon_{x x}-\varepsilon_{y y}\right)^{2}+\varepsilon_{x y}^{2} \mathrm{~d} V}} .
\end{aligned}
$$


For identification purposes, one advantage of isotropic elasticity over cubic elasticity appears in the previous system of equations : the identification of all elastic coefficients on each subdomain $\omega_{i}$ does not require the shear strain to be locally nonzero.

\subsubsection{Second variant : enforcement of measurements by kinematic penalization}

It is not necessarily desirable to impose exactly the kinematic measurements, particularly if they are corrupted by measurement noise. For this reason, it is interesting to consider the variant based on the functional

$$
F(\boldsymbol{v}, \boldsymbol{\tau}, \mathcal{B})=\alpha E(\boldsymbol{v}, \boldsymbol{\tau}, \mathcal{B})+\frac{\beta}{2}\|\boldsymbol{v}-\overline{\boldsymbol{u}}\|^{2},
$$

where $\alpha$ and $\beta$ are positive weighting coefficients [CAL 02]. In (10.19) the distance $\|\boldsymbol{v}-\overline{\boldsymbol{u}}\|$ must be defined such that the two terms of $F$ have comparable magnitudes. For example, $\|\boldsymbol{w}\|^{2}=E \int_{\Omega} \varepsilon[\boldsymbol{w}]: \varepsilon[\boldsymbol{w}] \mathrm{d} V$ if we focus on cases where $\mathcal{A}$ is isotropic (i.e. defined in terms of Young's modulus $E$ and Poisson's ratio $\nu$ ), or $\|\boldsymbol{w}\|^{2}=\gamma \boldsymbol{w} \cdot \boldsymbol{w}$ with a coefficient $\gamma$ to be chosen appropriately.

In line with previous work on CEG-based approaches for model updating, such as [REY 90, DER 02], the two partial minimizations are carried out successively. The first minimization (location step) gives $(\boldsymbol{u}, \boldsymbol{\sigma})$ with $\boldsymbol{\sigma} \in \mathcal{S}$, with $\mathcal{S}$ again defined by (10.11). Numerically, the stress fields are obtained using one of the previouslydiscussed representations. As with the first variant, the partial minimization of $E(\boldsymbol{v}, \boldsymbol{\tau}, \mathcal{B})$ with respect to $\mathcal{B}$ (correction step) is then explicit (provided that the coefficient $\beta$ does not depend on $\mathcal{B})$.

Functionals $F$ of the form 10.19 are convex over $\mathcal{C} \times \mathcal{S} \times \mathcal{A}$. As explained in [GEY 02, GEY 03], another family of functionals $\tilde{F}(\boldsymbol{v}, \boldsymbol{\tau}, \mathcal{S})$, separately convex over $\mathcal{C} \times \mathcal{S}$ and $\mathcal{A}$, can be defined from (10.19) in terms of the compliance tensor $\mathcal{S}=\mathcal{B}^{-1}$ rather than the stiffness tensor $\mathcal{B}$ :

$$
\tilde{F}(\boldsymbol{v}, \boldsymbol{\tau}, \mathcal{S})=F\left(\boldsymbol{v}, \boldsymbol{\tau}, \mathcal{S}^{-1}\right), \quad(\boldsymbol{v}, \boldsymbol{\tau}, \mathcal{S}) \in \mathcal{C} \times \mathcal{S} \times \mathcal{A} .
$$

\subsubsection{Comments}

Remark 1 The works [KOH 84, KOH 90] on conductivity imaging are based on a CEG-type functional adapted to electrostatic constitutive behavior. They also consider specific situations of overdetermined complete data on the boundary (potential and flux both known over the whole boundary $\partial \Omega$ ), and proceed by minimizing the energy gap between Dirichlet and Neumann solutions associated with a given conductivity field. A similar approach is followed for the identification of elastic moduli fields in [CON 95]. 
Remark 2 Functionals of the form (10.19) correspond to the modified CEG previously introduced for model updating (see references in §10.5). They have the advantage of being applicable to arbitrary, possibly quite scarce, data (introduced via the second term of (10.19)).

Remark 3 Reference [AVR 07] proposes an interpretation of the minimization of the CEG as a particular form of the virtual fields method.

Remark 4 The authors of [CHA 99], considering the problem of identification of heterogeneous moduli in terms of the classical minimization approach for a cost function $J(\boldsymbol{v})$ expressing the $L^{2}$-norm of the misfit between the experimental displacement data and their simulation $\boldsymbol{v}$ (i.e. defined as the second term of the functional (10.19)), proposed to include the constraint defined by the model equations in the form of a penalty via the sum $\mathcal{P}+\mathcal{P}^{\star}$ of the potential and complementary energies, whose optimal value (zero) is known beforehand. Given relation (10.5), this led them to propose the penalized formulation

$$
\mathcal{A}=\lim _{\eta \rightarrow 0} \underset{v \in \mathcal{C}, \tau \in \mathcal{S}, \mathcal{B} \in A}{\arg \min }\left(J(\boldsymbol{v})+\frac{1}{\eta} E(\boldsymbol{v}, \boldsymbol{\tau}, \mathcal{B})\right),
$$

wherein the functional used is equal (up to a multiplication factor and for a fixed value of the penalty parameter $\eta)$ to $F(\boldsymbol{v}, \boldsymbol{\tau}, \mathcal{B})$ as defined by (10.19). This observation thus gives a useful interpretation of the latter, and in particular of the compromise parameter $\beta$.

Remark 5 An original application of the CEG, proposed by [AND 06, AND 08], concerns data completion, i.e. the reconstruction of data on the boundary, given illposed boundary conditions of Cauchy type (simultaneous knowledge of displacements and tractions on part of the boundary, no boundary data on another part). An optimal control type of approach, based on the minimization of a CEG cost function (energy norm of the difference between Dirichlet and Neumann solutions) leads to a completion algorithm whose convergence is much faster than alternating methods such as that of Kozlov, Maz'ya, Fomin [KOZ 91].

\subsubsection{Some numerical examples}

The algorithm is illustrated by three examples; the first two are scalar problems while the last one is a vectorial case. The first two problems are solved with algorithm 1 with the functional $\tilde{F}$ defined by (10.20).

Example 1 (identification of a conductivity field). To test the performance of algorithm 1, we consider a scalar problem with "perfect measurements" on the unit square $\Omega=(0,1) \times(0,1)$ discretized with square elements. For instance, the solution to this conductivity problem is important in hydrology and has been solved in [KOH 88]. At the nodal points, the values of the measured field $u^{\star}=x+y+\frac{1}{3}\left(x^{3}+y^{3}\right)$, 
$f=-\operatorname{div}\left(\nabla u^{\star} / a_{\mathrm{ex}}\right)$ are assumed to be known, where $a_{\mathrm{ex}}=1 /\left(1+y^{2}\right)$ is the inverse of the conductivity. On the boundary, the given surface force is $g=\left(\partial_{n} u^{\star}\right) / a_{\mathrm{ex}}$. We assume that $\mathcal{E}=\mathcal{E}_{1}$ and $L_{1}(\boldsymbol{\sigma} . \mathbf{n}) \equiv \boldsymbol{\sigma} \cdot \mathbf{n}=g$ on all sides of the square $\Omega$. The observed convergence for the functional $\tilde{F}$ is $\left\|a^{n}-a_{\mathrm{ex}}\right\|_{\mathrm{L}^{2}} \sim O\left(h^{0.57}\right)$ and $\tilde{F}\left(\boldsymbol{\sigma}^{n}, a_{n}\right) \sim O\left(h^{1.16}\right)$, where $h$ is the characteristic diameter of a finite element. By applying the algorithm to $F$, the same type of convergence is obtained. Figure 10.1 shows plots of $\tilde{a}-a_{\mathrm{ex}}, a-a_{\mathrm{ex}}$ and $\tilde{a}-a$, where $\tilde{a}$ and $a$ respectively result from the minimization of $\tilde{F}$ and $F$.
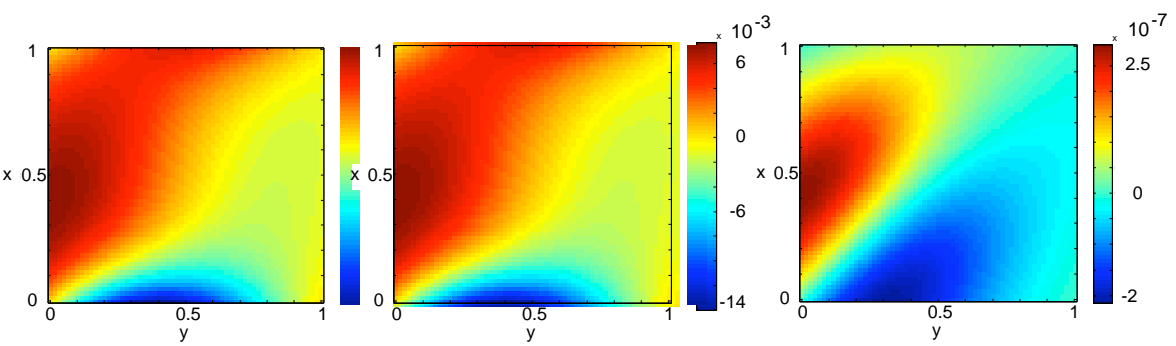

Figure 10.1. $a-a_{e x}$ (left), $\tilde{a}-a_{e x}$ (middle) et $a-\tilde{a}$ (right), computed using 400 quadrangular finite element, convergence threshold $\varepsilon_{c}=10^{-6}$

Example 2 (problem with a singularity). This second example tests the ability of algorithm 1 to capture local singularities or diffuse damage. Situations where the true conductivity inverse $a_{e x}$ is either $a_{e x}=1 /\left(\varepsilon+(y-1 / 2)^{2}\right)$, with $\varepsilon$ a positive constant (local singularity case), or $a_{e x}=1 /(1-D)\left(1+y^{2}\right)$, with $D \in(0,1)$ (diffuse damage case), are thus considered. As in example $1, \Omega$ is the unit square discretized with square elements. Algorithm 1 was found to require more iterations as $\varepsilon$ decreases, and in fact failed to converge for $\varepsilon \leq 0.005$ (cf. figure 10.2) ; improved results for $\varepsilon=0.005$ were obtained using a refined mesh. Figure 10.2 shows plots of $\tilde{a}-a_{\mathrm{ex}}, a-a_{\mathrm{ex}}$ and $\tilde{a}-a$. Finally, concerning the diffuse damage case, the number of iterations was found to increase as $D$ gets closer to 1 (10 iterations for $D=0,12$ for $D=0.5$ and 86 for $D=0.999)$.

Example 3 (Tensile test). A comparison of different identification methods has been undertaken as a joint effort by several research groups [AVR 08]. This comparison concerns in particular the results obtained in identifying elastic parameters in various experimental situations. The simplest of these situations is a simple tensile test on a 2024 aluminum alloy. The specimen is a bar of section $4.8 \times 4.8 \mathrm{~mm}$. Two elastic parameters are identified, Young's modulus $E$ and Poisson's ratio $\nu$. For this test, the displacement fields were provided by F. Hild of LMT Cachan. The images were taken by means of an 8 -bit camera $(1008 \times 1016$ pixels $)$ equipped with a long-distance microscope. A $2 \times 2 \mathrm{~mm}$ region of interest was observed. A series of 21 images was taken while an increasing load was applied to the specimen. In addition to the displacement fields and force measurements, reference elastic properties were provided. They were 


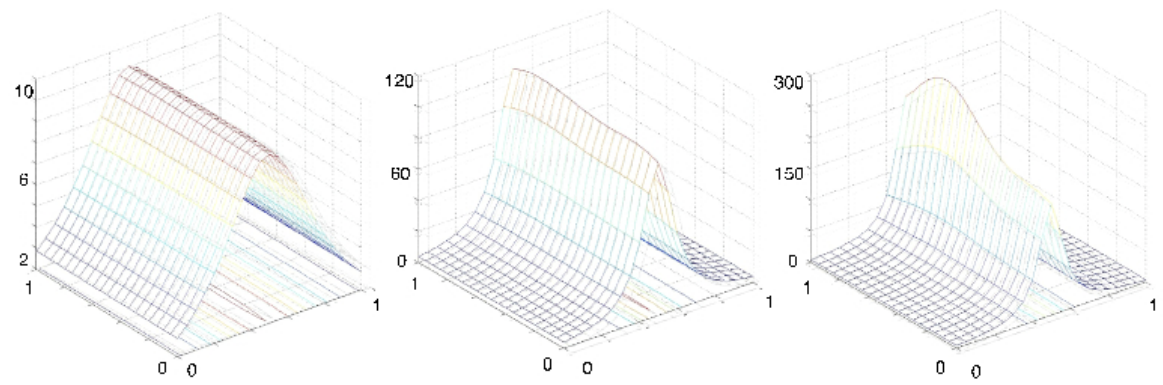

Figure 10.2. Identified values of $a_{e x}$ for different values of $\varepsilon: 0.1,0.01$ and 0.005 (400 quadrangular elements, convergence threshold $\varepsilon_{c}=10^{-6}$ )

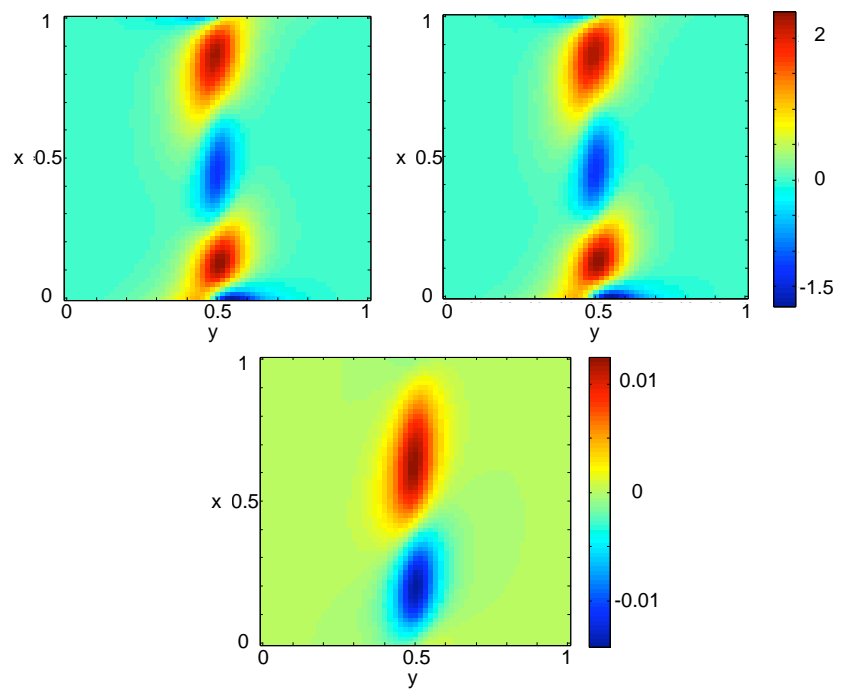

Figure 10.3. $a-a_{e x}$ (top left), $\tilde{a}-\tilde{a}_{e x}$ (top right) and $a-\tilde{a}$ (bottom), computed using 400 quadrangular elements, convergence threshold $\varepsilon_{c}=10^{-6}$

obtained by a linear regression on all the tensile tests using strain gage measurements, as $E_{\text {ref }}=76 \pm 0.5 \mathrm{GPa}, \nu_{\text {ref }}=0.33 \pm 0.01$.

For the last image, corresponding to the maximum (2003 N) of the applied load, the displacement field is shown in Figure 10.4. The components $u_{x}$ and $u_{y}$ respectively show the stretching of the bar and the contraction of its section. The fields are given at $15 \times 15$ points ; they are consistent with the identification method.

The results for the identified elastic parameters are given in Figure 10.5. For each image $i$, a set of parameters $\left(E_{i}, \nu_{i}\right)$ is identified, whose values are then compared 


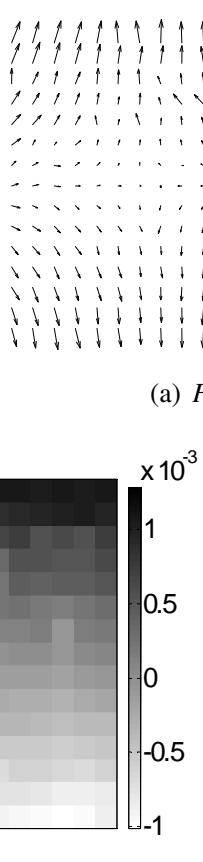

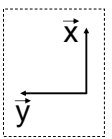

a) Field $\boldsymbol{u}$ (b) $u_{x}(\mathrm{~mm})$

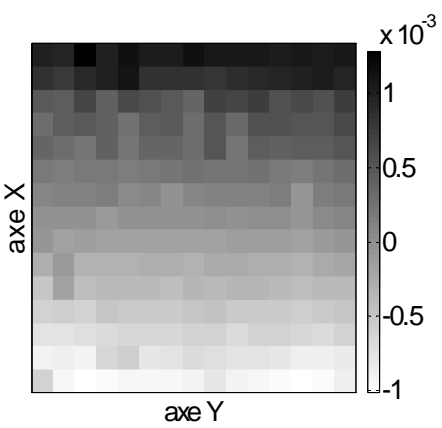

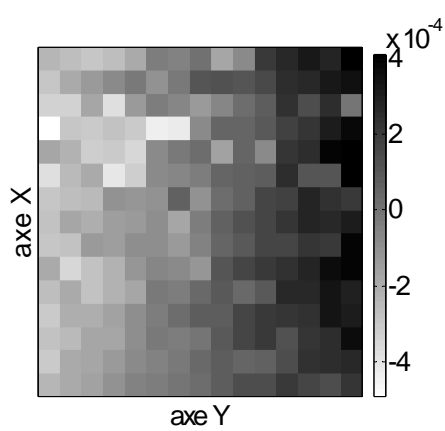

(c) $u_{y}(\mathrm{~mm})$

Figure 10.4. In-plane displacement components for $F=2003 \mathrm{~N}$

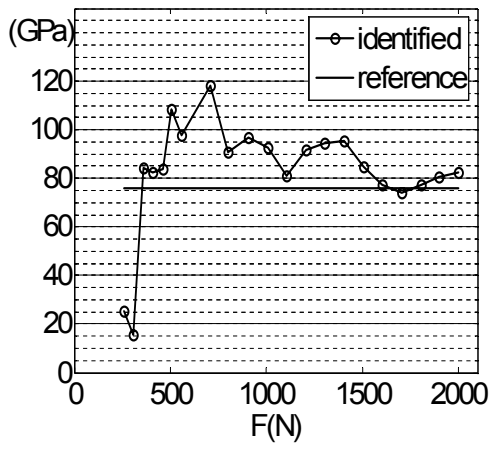

(a) $E$

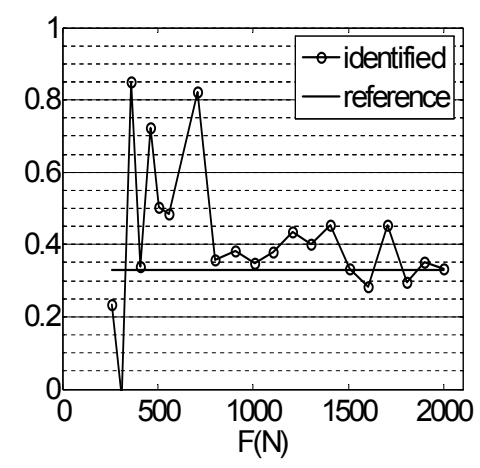

(b) $\nu$

Figure 10.5. Values of identified elastic coefficients

to the reference values. For low load levels, the identified parameters are not very close to the reference parameters, but the identification improves as the applied force increases. More generally, for low strain amplitudes (in the linear elastic range) the signal-to-noise ratio improves with the load level. 
By averaging the last 5 results $(i=17, \ldots, 21)$, the following identified values are obtained : $E_{i d}=78.3 \mathrm{GPa}, \nu_{i d}=0.343$. Comparing these values with the reference values, the relative discrepancies found are $3 \%$ for the Young modulus and $4 \%$ for the Poisson ratio. The obtained values are satisfactory and we see that it is advisable for this type of test to use a large number of image-force couples. Indeed, from one image to another, the identified elastic coefficients may vary significantly. This variability is associated here with experimental noise, which corrupts the data and hinders identification.

To conclude this test, homogeneous properties were identified from a simple tensile test. A comparison of different identification methods (in particular the virtual fields method and finite element method updating) was conducted as part of the benchmark organized by members of the GdR 2519 consortium. Identified values using other methods were in the 70-75 GPa range for the Young modulus, and in the 0.30.36 range for the Poisson ratio. Overall, a good agreement between the results obtained by all methods was thus reached. This experiment did not enable the demonstration of the main advantages of the identification method developed in this chapter, for instance its ability to yield both a stress field and a distribution of material properties. Situations that better exploit this potential are considered next.

\subsection{Extension to elastoplasticity}

\subsubsection{Formulation}

In this section, identification assumes elastic-plastic constitutive properties obeying the linear kinematic hardening model of Prager. Naturally, this simple model cannot reproduce all the complexity of the development of plasticity. It is expressed in terms of two plastic parameters : a yield stress $\sigma_{0}$ and a constant hardening modulus $k$.

The objective here is to develop a method to identify local parameters of this model and characterize its performance for identification. The use of models which are more representative of the actual behavior of metallic materials is possible, but will not be discussed in this section. The chosen model may be defined synthetically by the four equations :

$$
\begin{aligned}
\boldsymbol{\sigma} & =\mathcal{A}\left(\boldsymbol{\varepsilon}-\boldsymbol{\varepsilon}^{\mathrm{P}}\right), & & \dot{\boldsymbol{\varepsilon}}^{\mathrm{P}}=\dot{\gamma} \frac{\partial f}{\partial \boldsymbol{\sigma}}, \\
f(\boldsymbol{\sigma}, \boldsymbol{X}) & =(\boldsymbol{\sigma}-\boldsymbol{X})_{\mathrm{eq}}-\sigma_{0} \leq 0, \quad(10.21 \mathrm{~b}) & \dot{\boldsymbol{X}} & =\frac{2}{3} k \dot{\boldsymbol{\varepsilon}}^{\mathrm{P}},
\end{aligned}
$$

where $\mathcal{A}$ is the elasticity tensor, $\varepsilon^{\mathrm{p}}$ the plastic part of the strain tensor, $\gamma$ the plastic multiplier, $f$ the yield function and $\boldsymbol{X}$ the backstress tensor.

The above-described Prager model can be recast in incremental form using an implicit time discretization [SIM 98]. In what follows, subscripts $n$ and $n+1$ indicate 
values at the initial and final instants of the current, $n+1$-th, time step. For a plastic step for which the initial hardening is zero (i.e. $\boldsymbol{X}_{n}=0$ and $\varepsilon_{n}^{\mathrm{p}}=0$ ), we can establish an explicit formula for the elastoplastic secant tensor $\mathcal{A}_{p}^{s}$ [SIM 98] :

$$
\boldsymbol{\sigma}_{n+1}=\left[\mathcal{A}_{p}^{s}\right]_{n+1}: \boldsymbol{\varepsilon}_{n+1}, \quad\left[\mathcal{A}_{p}^{s}\right]_{n+1}=\left[\mathcal{A}^{-1}-\frac{3 \Delta \gamma\left(\sigma_{0}\right)}{3+2 k \Delta \gamma\left(\sigma_{0}\right)} \boldsymbol{P}\right]^{-1}
$$

where $\Delta \gamma$ is the plastic multiplier increment and $\boldsymbol{P}$ is a mapping matrix. In other cases, the relationship between $\boldsymbol{\sigma}_{n+1}$ and $\varepsilon_{n+1}$ is not available in explicit form, but an elastoplastic tangent tensor $\mathcal{A}_{p}^{t}$ can be defined such that $\left[\mathcal{A}_{p}^{t}\right]_{n+1}=\mathrm{d} \boldsymbol{\sigma} /\left.\mathrm{d} \varepsilon\right|_{n+1}$.

We will consider two kinds of formulation for the mechanical problem : a standard formulation and an incremental formulation. The former is associated with a global load step for which the strain is obtained between an undeformed reference state and a deformed state. The latter is associated with a load increment for which the strain is computed between two successive deformed states.

The elastic identification problem thus consists of finding the elasticity tensor $\mathcal{A}^{s}$ and the stress $\boldsymbol{\sigma}$ which satisfy the equilibrium equation (10.24a), the constitutive equation (10.24b) and the global equilibrium (10.24c) for a load step during which no hardening occurs. Plastic identification consists of finding, during a plastic evolution, the elastoplastic tangent stiffness tensor $\mathcal{A}_{p}^{t}$ and the stress increment $\Delta \sigma$ so as to verify the governing equations (10.25a), 10.25b and (10.25c) of the incremental problem, applied to a plastic increment :

Standard formulation :

$$
\begin{array}{lll}
\operatorname{div} \boldsymbol{\sigma}=0 & \text { dans } \Omega, & (10.24 \mathrm{a}) \\
\boldsymbol{\sigma}=\mathcal{A}^{s}: \varepsilon[\overline{\boldsymbol{u}}] & \text { dans } \Omega, & (10.24 \mathrm{~b}) \\
L_{i}(\boldsymbol{\sigma} \boldsymbol{n})=\boldsymbol{g}_{i} & \operatorname{sur} \Gamma_{i} . & (10.24 \mathrm{c})
\end{array}
$$

Within the elastic domain, the behavior is described by an elastic stiffness tensor $\mathcal{A}$, which can be obtained from either the "standard formulation" or the "incremental formulation". In theory, $\mathcal{A}=\mathcal{A}^{s}=\mathcal{A}^{t}$. In practice, in order to use data for identification with an optimal signal-to-noise ratio, a secant formulation for the identification of elastic parameters is chosen.

Similarly to what was done for the elastic case, it is possible to write the CEG $E\left(\overline{\boldsymbol{u}}, \boldsymbol{\tau}, \mathcal{B}^{s}\right)$ for the standard formulation, and the CEG $E\left(\overline{\boldsymbol{u}}, \Delta \boldsymbol{\tau}, \mathcal{B}^{t}\right)$ for the incremental formulation. Stress and stress increment fields respectively belong to spaces $\mathcal{S}$ and $\Delta \mathcal{S}$, defined similarly to (10.6b). The results given in Proposition 1 are still valid in both cases. 


\subsubsection{Numerical method}

The minimization of the CEG functional with respect to stress fields is carried out analogously to the elastic case [LAT 07, LAT 08]. The computation of material property distributions remains to be addressed.

\subsubsection{Plastic detection}

Given the impossibility of identifying locally more than three parameters simultaneously, the identification of elastic parameters and plastic parameters are performed separately, with plastic parameters identified only after the elastic parameters are determined. The first loading step must therefore be assumed to be purely elastic. The elastic parameters identified are then considered as "reference" parameters, denoted $\mathcal{A}^{r}$. Among the following loading steps, a distinction must be made between purely elastic and plastic steps. To detect that a step is plastic, elastic identification is performed, in order to compare the elastic tensor $\mathcal{A}$ thus obtained with the reference elastic tensor $\mathcal{A}^{r}$. The step is then deemed plastic whenever this difference is too large. It is possible to compare tensors obtained after either the complete loading or just one load increment.

\subsubsection{Computation of plastic material properties}

Concerning the determination of plastic material properties, two cases may arise depending on whether a secant or tangent problem is addressed. Both situations also involve the elastic parameters. Both types of plastic problem are described next, aiming at the determination of two plastic unknowns : the hardening modulus $k$ and the yield stress $\sigma_{0}$.

Secant problem.. For the secant problem, we seek to determine the elastoplastic secant tensor $\mathcal{B}_{p}^{s}$, which is possible when the backstress tensor $\boldsymbol{X}_{n}$ is zero :

$$
\left[\mathcal{B}_{p}^{s}\right]_{n+1}=\left[\mathcal{A}^{-1}-\frac{3 \Delta \gamma\left(\sigma_{0}\right)}{3+2 k \Delta \gamma\left(\sigma_{0}\right)} \boldsymbol{P}\right]^{-1} .
$$

Just as with the elastic tensor, the plastic secant tensor can be written (assuming plane stress conditions) in a form similar to the elastic tensor :

$$
\mathcal{B}_{p}^{s}=\left[\begin{array}{ccc}
b_{p s 1} & b_{p s 2} & 0 \\
b_{p s 2} & b_{p s 1} & 0 \\
0 & 0 & b_{p s 3}
\end{array}\right] .
$$

Equation (10.26) allows each of the three independent entries of $\mathcal{B}_{p}^{s}$ to be written in terms of $k$ and $\sigma_{0}$. Upon diagonalizing $\mathcal{B}_{p}^{s}$ and enforcing the stationarity of $E(\overline{\boldsymbol{u}}, \boldsymbol{\tau})$ with respect to the eigenvalues of $\mathcal{B}_{p}^{s}$, the three equations obtained involve a plastic variable $K_{p}\left(k, \sigma_{0}, \boldsymbol{\tau}\right)$. The induced coupling between $k$ and $\sigma_{0}$ thus does not allow their simultaneous determination by considering only the secant problem associated with the first plastic step. 
Tangent problem.. The tangent problem can be written for a plastic loading increment, knowing the backstress $\boldsymbol{X}_{n}$ computed at the previous step. For incremental plasticity, the elastoplastic tangent tensor is given by (10.23). The expression of this tensor is not simple enough to provide the proof that the three plasticity equations are dependent. However, in simple situations (e.g. uniaxial tension), a numerical calculation enables us to plot the functional associated with the tangent problem, whose minimization yields the sought plastic parameters (see Figure 10.6 for the case of a tensile test on a steel sample whose properties are $E=200 \mathrm{GPa}, \nu=0.3, k=10$ $\mathrm{GPa}$ and $\sigma_{0}=300 \mathrm{MPa}$ ). For this situation, the functional has an elongated valley which prevents the identification of the parameter $\sigma_{0}$, whereas the hardening modulus $k$ can be identified.

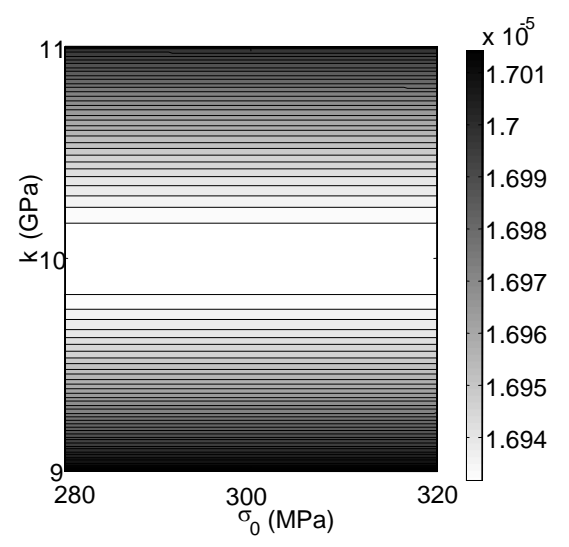

Figure 10.6. Values of the functional $E\left(\overline{\boldsymbol{u}}, \Delta \boldsymbol{\tau}, \mathcal{B}^{t}\right)$ for a tensile test

To identify both coefficients $k$ and $\sigma_{0}$, it is thus necessary to consider two successive plastic loading steps, denoted $n$ and $n+1$. Both steps are schematically depicted in Figure 10.7, with the tangent and secant moduli used for each step. The first plastic step $n$ is best exploited by means of a secant problem, which allows larger strains to be considered than for the tangent problem, and thus to enjoy a better signal-to-noise ratio for identification purposes. Since the yield threshold is reached during this first step, the yield stress $\sigma_{0}$ is to be identified from a secant formulation associated with the CEG functional $E_{s}$ defined by

$$
E_{s}\left(\overline{\boldsymbol{u}}, \boldsymbol{\tau}, \sigma_{0}, k\right)=E\left(\overline{\boldsymbol{u}}, \boldsymbol{\tau}, \mathcal{B}_{p}^{s}\left(\boldsymbol{\tau}, \sigma_{0}, k\right)\right) .
$$

Then the second plastic step $n+1$ is used to identify the hardening modulus $k$, a tangent problem associated with the CEG functional $E_{t}$ defined by :

$$
E_{t}\left(\overline{\boldsymbol{u}}, \Delta \boldsymbol{\tau}, \sigma_{0}, k\right)=E\left(\overline{\boldsymbol{u}}, \Delta \boldsymbol{\tau}, \mathcal{B}_{p}^{t}\left(\boldsymbol{\tau}, \sigma_{0}, k\right)\right) .
$$




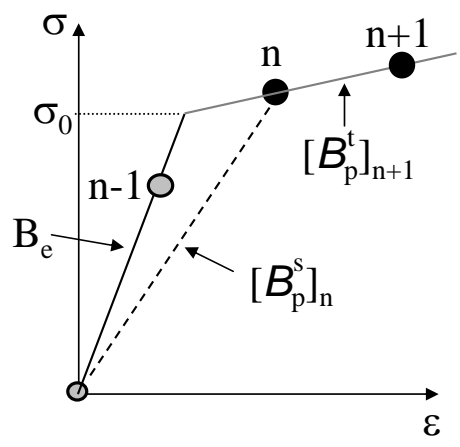

Figure 10.7. Curve $\sigma-\varepsilon$

The fact that the tangent and secant elastoplastic tensors depend on the stress tensor (or stress increment) somewhat complicates the resulting algorithm, given next.

Algorithm 2 Two-step plastic algorithm, with enforce kinematic measured $\overline{\boldsymbol{u}}$ :

- Plastic initialization of the algorithm

$\sigma_{0}^{0}=\left(\sigma_{n-1}\right)_{I I}$

$k^{0}$ estimated from an elastic tangent computation

$E_{t}\left(\overline{\boldsymbol{u}}, \Delta \boldsymbol{\sigma}_{n}, \sigma_{0}^{0}, k^{0}\right) \leq E_{t}\left(\overline{\boldsymbol{u}}, \Delta \boldsymbol{\tau}, \sigma_{0}^{0}, k^{0}\right), \quad \forall \Delta \boldsymbol{\tau} \in \Delta \mathcal{S}$

- (For known $\left.\boldsymbol{\sigma}_{n}^{i}, \Delta \boldsymbol{\sigma}_{n+1}^{i}, \sigma_{0}^{i}, k^{i}\right)$, successively determine $\left(\boldsymbol{\sigma}_{n}^{i+1}, \Delta \boldsymbol{\sigma}_{n+1}^{i+1}, \sigma_{0}^{i+1}, k^{i+1}\right)$ by solving :

step 1 : plastic computation step $n+1$, incremental formulation

$$
\begin{aligned}
& E_{t}\left(\overline{\boldsymbol{u}}, \Delta \boldsymbol{\sigma}_{n+1}^{i+1}, \sigma_{0}^{i}, k^{i}\right) \leq E_{t}\left(\overline{\boldsymbol{u}}, \Delta \boldsymbol{\tau}, \sigma_{0}^{i}, k^{i}\right), \quad \forall \Delta \boldsymbol{\tau} \in \Delta \mathcal{S} \\
& E_{t}\left(\overline{\boldsymbol{u}}, \Delta \boldsymbol{\sigma}_{n+1}^{i+1}, \sigma_{0}^{i}, k^{i+1}\right) \leq E_{t}\left(\overline{\boldsymbol{u}}, \Delta \boldsymbol{\sigma}_{n+1}^{i+1}, \sigma_{0}^{i}, \kappa\right), \quad \forall \kappa
\end{aligned}
$$

step 2 : plastic computation step $n$, standard formulation

$$
\begin{aligned}
& E_{s}\left(\overline{\boldsymbol{u}}, \boldsymbol{\sigma}_{n}^{i+1}, \sigma_{0}^{i}, k^{i+1}\right) \leq E_{s}\left(\overline{\boldsymbol{u}}, \boldsymbol{\tau}, \sigma_{0}^{i}, k^{i+1}\right), \quad \forall \boldsymbol{\tau} \in \mathcal{S} \\
& E_{s}\left(\overline{\boldsymbol{u}}, \boldsymbol{\sigma}_{n}^{i+1}, \sigma_{0}^{i+1}, k^{i+1}\right) \leq E_{s}\left(\overline{\boldsymbol{u}}, \boldsymbol{\sigma}_{n}^{i+1}, s_{0}, k^{i+1}\right), \quad \forall s_{0}
\end{aligned}
$$

- Convergence test.

\subsection{Formulations based on the Legendre-Fenchel transform}

The formulation developed in Section 10.3 for elastoplastic identification is based on an incremental version of the linear elastic CEG. A generalization to non-linear behavior of the linear elastic CEG, which is more consistent from a theoretical point of view but so far seldom exploited for identification purposes, exploits the LegendreFenchel transform. For example, for non-linear elastic behavior defined by the convex 
free energy density $\psi(\varepsilon)$, we set

$$
E(\boldsymbol{v}, \boldsymbol{\tau}, \psi)=\int_{\Omega}\left(\psi(\boldsymbol{\varepsilon}[\boldsymbol{v}])+\psi^{\star}(\boldsymbol{\tau})-\boldsymbol{\tau}: \varepsilon[\boldsymbol{v}]\right) \mathrm{d} V,
$$

where the potential $\psi^{\star}(\boldsymbol{\tau})$, given by

$$
\psi^{\star}(\boldsymbol{\tau})=\sup _{\boldsymbol{\varepsilon}}(\boldsymbol{\tau}: \varepsilon-\psi(\varepsilon)),
$$

is the complementary energy density, i.e. the Legendre-Fenchel transform of $\psi(\varepsilon)$. Defining the Legendre-Fenchel gap $e(\varepsilon, \tau)$ by

$$
e(\varepsilon, \boldsymbol{\tau})=\psi(\varepsilon)+\psi^{\star}(\boldsymbol{\tau})-\boldsymbol{\tau}: \varepsilon,
$$

classical convex analysis results yield

$$
e(\boldsymbol{\varepsilon}, \boldsymbol{\tau}) \geq 0 \text { (pour tous } \varepsilon, \boldsymbol{\tau}) ; e(\varepsilon, \boldsymbol{\tau})=0 \Longleftrightarrow \boldsymbol{\tau}=\frac{\partial \psi}{\partial \boldsymbol{\varepsilon}} .
$$

The choice made in (10.30) to use $e(\varepsilon[\boldsymbol{v}], \boldsymbol{\tau})$ as CEG density is thus fully justified, the latter quantity being non-negative and vanishing when $\tau$ and $\varepsilon$ are linked by the (nonlinear elastic) constitutive model. Of course, choosing the potential $\psi(\varepsilon)$ as quadratic and convex gives the linear elastic CEG (10.4).

The definition of the CEG functional using the Legendre-Fenchel density can be generalized to standard generalized constitutive models [HAL 75, GER 83], defined by means of a free energy density $\psi$ and a dissipation potential $\varphi$. This approach is used in [MOË 99] for error estimation in finite element calculations in non-linear conditions. The CEG functional associated with a standard generalized elastic-plastic model is defined by

$$
\begin{aligned}
E\left(\boldsymbol{v}, \varepsilon^{\mathrm{p}}, p, \boldsymbol{\tau}, R ; \boldsymbol{\theta}\right) & =\int_{\Omega}\left\{\left[\psi\left(\varepsilon[\boldsymbol{v}]-\varepsilon^{\mathrm{p}}, p\right)+\psi^{\star}(\boldsymbol{\tau}, R)-\boldsymbol{\tau}:\left(\varepsilon[\boldsymbol{v}]-\varepsilon^{\mathrm{P}}\right)\right]_{t=T}\right. \\
& \left.+\int_{0}^{T}\left[\varphi\left(\varepsilon^{\mathrm{P}}, \dot{p}\right)+\varphi^{\star}(\sigma, R)-\boldsymbol{\tau}: \dot{\boldsymbol{\varepsilon}}^{P}+R \dot{p}\right] \mathrm{d} t\right\} \mathrm{d} V,
\end{aligned}
$$

where $\varepsilon^{\mathrm{P}}$ is the plastic part of the strain, $p$ is the cumulative plastic strain, $R$ is the yield stress, and $\boldsymbol{\theta}$ is a vector of (possibly heterogeneous) constitutive parameters.

The identification of $\boldsymbol{\theta}$ can then be formulated as

$$
\boldsymbol{\vartheta}=\underset{\theta}{\arg \min } \mathcal{E}(\boldsymbol{\theta}),
$$

where $\mathcal{E}(\boldsymbol{\theta})$ is defined by a partial minimization of the CEG functional (10.33) :

$$
\mathcal{E}(\boldsymbol{\theta})=\min _{\left(v, \varepsilon^{P}, \dot{p}\right) \in \mathcal{C}^{\mathrm{EP}},(\tau, R) \in \mathcal{S}^{\mathrm{EP}}} E\left(\boldsymbol{v}, \boldsymbol{\varepsilon}^{\mathrm{P}}, \dot{p}, \boldsymbol{\tau}, R ; \boldsymbol{\theta}\right)
$$


and the admissible spaces $\mathcal{C}^{\mathrm{EP}}, \mathcal{S}^{\mathrm{EP}}$ are defined in terms of the spaces $\mathcal{C}$ and $\mathcal{S}$ of kinematically and statically admissible fields by

$$
\begin{aligned}
& \mathcal{C}^{\mathrm{EP}}=\left\{\boldsymbol{v}, \boldsymbol{\varepsilon}^{\mathrm{P}}, p \mid \boldsymbol{v} \in \mathcal{C}, \boldsymbol{\varepsilon}^{\mathrm{P}}: \mathbf{1}=0, \sqrt{(2 / 3) \varepsilon^{\mathrm{p}}: \varepsilon^{\mathrm{p}}}=\dot{p}\right\}, \\
& \mathcal{S}^{\mathrm{EP}}=\left\{\boldsymbol{\tau}, R \mid \boldsymbol{\tau} \in \mathcal{S},\|\boldsymbol{\tau}\|_{\mathrm{eq}}-R-R_{0} \leq 0\right\} .
\end{aligned}
$$

For isotropic elastic-plastic behavior defined by an initial yield stress $R_{0}$, the von Mises criterion, an associated flow rule and linear isotropic hardening $h$, we have $\boldsymbol{\theta}=\left(\mu, \nu, R_{0}, h\right)$ and the CEG functional is given (with $\mathcal{A}=\mathcal{A}(\mu, \nu)$ and $\varepsilon^{\mathrm{E}}[\boldsymbol{v}]=$ $\left.\varepsilon[v]-\varepsilon^{\mathrm{P}}\right)$ by

$$
\begin{aligned}
E\left(\boldsymbol{v}, \boldsymbol{\varepsilon}^{\mathrm{P}}, p, \boldsymbol{\tau}, R ; \boldsymbol{\theta}\right)= & \int_{\Omega}\left\{\left[\frac{1}{2}\left(\boldsymbol{\tau}-\mathcal{A}: \varepsilon^{\mathrm{E}}[\boldsymbol{v}]\right): \mathcal{A}^{-1}:\left(\boldsymbol{\tau}-\mathcal{A}: \varepsilon^{\mathrm{E}}[\boldsymbol{v}]\right)\right.\right. \\
& \left.\left.+\frac{1}{2 h}(R-h p)^{2}\right]_{t=T}+\int_{0}^{T}\left[R_{0} \dot{p}-\boldsymbol{\sigma}: \dot{\boldsymbol{\varepsilon}}^{\mathrm{P}}+R \dot{p}\right] \mathrm{d} t\right\} \mathrm{d} V .
\end{aligned}
$$

A different approach, also based on a gap built from the Fenchel-Legendre transform, is proposed in [HAD 07a, HAD 07b] to address situations where overdetermined measurements of the boundary are available. The proposed functional measures the difference between two solutions, defined for the same constitutive model and respectively associated with kinematic or static boundary data. The authors have primarily focused on the identification of viscoelastic constitutive parameters. The numerical implementation of this approach demonstrates in particular the improvement of local convexity (in the constitutive parameter space) of the proposed functional compared to a least squares functional measuring the boundary displacement misfit.

\subsection{Suitable formulations for dynamics or vibration}

Applications of the concept of constitutive equation gap to identification problems initially focused on model updating using vibrational data (experimental information on natural frequencies and modal displacements, possibly after processing the measured time response). This is partly motivated by the fact that many structures in operational conditions are subjected to dynamic loading or vibrations. Early work in this direction, carried out at LMT (ENS Cachan), includes the thesis [REY 90].

Formulation in the frequency domain. Model updating using the CEG in the framework of conservative dynamic vibrations is typically based on a functional of the form

$$
\mathcal{E}(\mathcal{A}, \rho)=\min _{v, \tau, \gamma} \sum_{\hat{\omega} \text { mesuré }}\left\{E_{\hat{\omega}}(\boldsymbol{v}, \boldsymbol{\tau}, \boldsymbol{\gamma}, \mathcal{A}, \rho)+\frac{\beta}{2} \int_{D} a(\boldsymbol{v}-\overline{\boldsymbol{u}}, \boldsymbol{v}-\overline{\boldsymbol{u}}) \mathrm{d} V\right\},
$$


defined by the partial minimization of a modified CEG functional

$$
\begin{aligned}
E_{\hat{\omega}}(\boldsymbol{v}, \boldsymbol{\tau}, \boldsymbol{\gamma}, \mathcal{A}, \rho)=\frac{\alpha}{2} \int_{\Omega}(\boldsymbol{\tau}-\mathcal{A}: \varepsilon[\boldsymbol{v}]): \mathcal{A}^{-1}:(\boldsymbol{\tau}-\mathcal{A}: \varepsilon[\boldsymbol{v}]) \mathrm{d} V \\
+\frac{1-\alpha}{2} \int_{\Omega} \frac{1}{\rho \hat{\omega}^{2}}\left\|\boldsymbol{\gamma}+\rho \hat{\omega}^{2} \boldsymbol{v}\right\|^{2} \mathrm{~d} V
\end{aligned}
$$

This functional in fact considers two constitutive equations : relation $\sigma=\mathcal{A}: \varepsilon$ of linear elasticity, and $\gamma=-\rho \omega^{2} \boldsymbol{u}$, linking the acceleration quantity density $\gamma$ to the displacement $\boldsymbol{u}$. The dynamic admissibility constraint reads, in weak form :

$$
\mathcal{R}(\boldsymbol{\tau}, \boldsymbol{\gamma} ; \boldsymbol{w}):=\int_{\Omega}(\boldsymbol{\tau}: \boldsymbol{\varepsilon}[\boldsymbol{w}]+\boldsymbol{\gamma} \cdot \boldsymbol{w}) \mathrm{d} V=0 \quad(\forall \boldsymbol{w} \in \mathcal{V})
$$

and can be combined with $E_{\hat{\omega}}(\boldsymbol{v}, \boldsymbol{\tau}, \boldsymbol{\gamma}, \mathcal{A}, \rho)$ using a Lagrangian $\mathcal{L}=E_{\hat{\omega}}-\mathcal{R}$. The stationarity equations for $\mathcal{L}$ are

$$
\begin{array}{lr}
0=\alpha \int_{\Omega} \varepsilon[\boldsymbol{w}]: \mathcal{A}: \varepsilon[\tilde{\boldsymbol{u}}] \mathrm{d} V-\int_{\Omega} \rho \hat{\omega}^{2} \boldsymbol{w} \tilde{\boldsymbol{u}} \mathrm{d} V+\beta \int_{D} a(\boldsymbol{u}-\overline{\boldsymbol{u}}, \tilde{\boldsymbol{u}}) \mathrm{d} V & (\forall \tilde{\boldsymbol{u}} \in \mathcal{V}), \\
0=\int_{\Omega}\left[\boldsymbol{\varepsilon}\left[\boldsymbol{u}-\frac{1}{\alpha} \boldsymbol{w}\right]: \mathcal{A}: \boldsymbol{\varepsilon}[\tilde{\boldsymbol{w}}]-\rho \hat{\omega}^{2}\left(\boldsymbol{u}+\frac{1}{(1-\alpha)} \boldsymbol{w}\right) \tilde{\boldsymbol{w}}\right] \mathrm{d} V & (\forall \tilde{\boldsymbol{w}} \in \mathcal{V}), \\
\boldsymbol{\sigma}=\mathcal{A} \varepsilon\left[\boldsymbol{u}-\frac{1}{\alpha} \boldsymbol{w}\right], & (10.35 \mathrm{~b}) \\
\left.\boldsymbol{\gamma}=-\rho \hat{\omega}^{2}\left[\boldsymbol{u}+\frac{1}{(1-\alpha)} \boldsymbol{w}\right] .35 \mathrm{c}\right) & \text { (10.35d) }
\end{array}
$$

Equations (10.35a), (10.35b) lead to a coupled system for the unknowns $\boldsymbol{u}, \boldsymbol{w}$, with $\boldsymbol{\sigma}, \boldsymbol{\gamma}$ given explicitly by (10.35a), (10.35b). The CEG densities associated with the elastic and kinetic constitutive relations are given by

$$
\frac{1}{\alpha} \varepsilon[\boldsymbol{w}]: \mathcal{A}: \varepsilon[\boldsymbol{w}], \quad \rho\|\boldsymbol{w}\|^{2} .
$$

Many numerical experiments [REY 90, BEN 95, LAD 99, BUI 00, DER 01, DER 02] have emphasized an important property of CEG densities, namely their tendency to reach their largest values in "incorrectly modeled areas" whose material properties present a discrepancy $\Delta \rho, \Delta \mathcal{A}$ with respect to the assumed values $\mathcal{A}$, $\rho$, especially if their spatial size is small compared to the analyzed structure (defects).

Concerning the identification of constitutive parameters under dynamic conditions, a "relaxed" version of the constitutive equation gap has been more recently proposed for transient dynamic conditions [FEI 06, NGU 08]. The idea is to avoid the exact enforcement (via admissible spaces $\mathcal{C}, \mathcal{S}$ ) of kinematic and dynamic data, which may be 
very noisy in dynamic tests. With reference to e.g. Hopkinson split bar dynamic testing, simultaneous experimental knowledge of all forces and velocities on the sample boundary is assumed. The principle of this relaxed approach (explained here in a static framework for simplicity) is then based on the definition of $\mathcal{C}, \mathcal{S}$ in terms of fictitious data (displacements and forces) $\boldsymbol{\xi}, \varphi$ :

$$
\begin{aligned}
\mathcal{C}(\boldsymbol{\xi}, \partial \Omega) & =\left\{\boldsymbol{v} \mid v_{i} \in \mathrm{H}^{1}(\Omega), \boldsymbol{v}=\boldsymbol{\xi} \text { sur } \partial \Omega\right\}, \\
\mathcal{S}(\boldsymbol{\varphi}, \partial \Omega) & =\left\{\boldsymbol{\tau} \in \mathrm{H}_{\mathrm{div}}(\Omega), \boldsymbol{\tau} . \boldsymbol{n}=\boldsymbol{\varphi} \text { sur } \partial \Omega\right\} .
\end{aligned}
$$

Considering for clarity the identification of elastic moduli, a modified CEG functional $\mathcal{H}(\mathcal{A})$ is defined as

$$
\mathcal{H}(\mathcal{A})=\min _{(\xi, \varphi)}\left(\min _{(v \in \mathcal{C}(\xi, \partial \Omega), \tau \in \mathcal{S}(\varphi, \partial \Omega))} H(\boldsymbol{v}, \boldsymbol{\tau}, \boldsymbol{\xi}, \boldsymbol{\varphi}, \mathcal{A})\right)
$$

with

$$
\begin{aligned}
H(\boldsymbol{v}, \boldsymbol{\tau}, \boldsymbol{\xi}, \boldsymbol{\varphi}, \mathcal{A})=\frac{1}{2} \int_{\Omega}(\boldsymbol{\tau}-\mathcal{A}: \varepsilon[\boldsymbol{v}]) & : \mathcal{A}^{-1}:(\boldsymbol{\tau}-\mathcal{A}: \varepsilon[\boldsymbol{v}]) \mathrm{d} V \\
& +\int_{S}\left(\frac{A}{2}\|\overline{\boldsymbol{u}}-\boldsymbol{\xi}\|_{U}^{2}+\frac{B}{2}\|\overline{\boldsymbol{T}}-\boldsymbol{\varphi}\|_{T}^{2}\right) \mathrm{d} S
\end{aligned}
$$

Coefficients $A$ and $B$ ensure the dimensional consistency of the functional $\mathcal{H}$ and enable the two types of data to be weighted according to their expected quality. This approach implicitly performs a smoothing of noisy data $\overline{\boldsymbol{u}}, \overline{\boldsymbol{T}}$. Numerical experiments in dynamic identification [FEI 06, NGU 08] have shown that this approach can operate satisfactorily for high levels of data noise, for which the performance of other functionals (including least squares misfit functionals acting directly on the boundary data) markedly deteriorates.

\subsection{Conclusions}

The identification of parameters featured in constitutive laws is an interesting and nontrivial problem. Indeed, the experimentalist measures certain "outputs" corresponding to certain "inputs". Before the advent of kinematic field measurements, experiments were generally designed in such a way that the mechanical state could be assumed homogeneous in the region of interest where gauges are applied. For homogeneous isotropic materials, such experiments allow the identification of the Young modulus and of the Poisson ratio. For general homogeneous anisotropic materials the identification of all elastic constants needs a more sophisticated analysis.

Over the last two decades, completely new experimental techniques enabling the measurement of displacement fields have appeared. Such techniques are particularly 
interesting when multiaxial loading along two or three perpendicular directions is applied and strain field heterogeneity occurs. Indeed, in such situations, a displacement field measurement is needed to check and quantify the heterogeneity of the strain field. The identification methods presented in this chapter use the vast amount of information contained in these field measurements. We have particularly emphasized the identification problem in heterogeneous linear elastic conditions, illustrating this approach with both academic examples and a case study with available experimental data. The identification algorithm used has been presented and discussed in detail to facilitate its subsequent use by the reader. An interesting aspect of local identification is the study of the sensitivity to contrasts of material properties. As expected, errors in the identification increase with the contrast, because of strong gradients induced in the stress and strain fields, which must then be approximated with the highest possible accuracy by the identification method.

The CEG elastic identification procedure also enables us to establish energy balances in polycyclic fatigue. Stress fields are identified over a mechanical cycle so as to estimate the mechanical energy locally supplied during a fatigue cycle. The originality of this method is to consider small variations in a secant elastoplastic stiffness tensor, mainly due to microplasticity mechanisms, as small variations in an elastic stiffness tensor. The latter is determined for each loading step during the mechanical cycle, and locally since no assumption is made about constitutive homogeneity. The spatial and temporal variability of material behavior is described as accurately as possible. The method allows the identification of stress fields associated with a heterogeneous material. A preliminary study involving this type of energy balance in the context of fatigue is presented in Chapter 16.

Kinematic measurements using digital image correlation enable data to be obtained on in-plane displacement fields. CEG-based identification methods have therefore so far been mostly developed for configurations for which the assumption of plane stress can be applied, the material properties also being identified in a twodimensional domain. The identification of three-dimensional heterogeneous properties is an interesting extension of the methods presented in this chapter. Kinematic field measurements in the entire volume are in fact made possible by tomography techniques, which are beginning to be used for mechanical tests but remain difficult to implement. Meanwhile, it is also important to note that the CEG (especially in its "modified" form) can be used with data obtained using other measurement methods.

Finally, the case of elastoplasticity has been considered using different CEG-based approaches. The first approach, an incremental version of the linear elastic CEG, raises some issues when the plastic zones evolve rapidly during loading. In an interesting extension of the identification method, the identification of hardening laws with more parameters, such as those involving an exponential term accounting for the observed saturation of hardening steels, could be considered. The second approach, based on the Legendre-Fenchel transformation, should help to overcome these difficulties. It is 
more consistent from a theoretical standpoint, but its practical implementation remains to be achieved. The formalism and the underlying theoretical framework should give rise to yet other interesting generalizations of the concept of CEG.

\subsection{Bibliographie}

[AND 92] ANDRIEUX S., BEN ABDA A., «Identification de fissures planes par une donnée au bord unique ; un procédé direct de localisation et d'identification. », Comptes Rendus Mécanique, vol. 315, p. 1323-1328, 1992.

[AND 96] ANDRIEUX S., BEN ABDA A., «Identification of planar cracks by complete overdetermined data : inversion formulae. », Inverse Problems, vol. 12, p. 553-563, 1996.

[AND 06] Andrieux S., Baranger T., Ben AbDa A., «Solving Cauchy problems by minimizing an energy-like functional », Inverse Problems, vol. 22, p. 115-133, 2006.

[AND 08] ANDRIEUX S., BARANGER T., «An energy error-based method for the resolution of the Cauchy problem in 3D linear elasticity», Comp. Meth. Appl. Mech. Eng., vol. 197, p. 902-920, 2008.

[AVR 07] AVRIL S., PIERron F., «General framework for the identification of constitutive parameters from full-field measurements in linear elasticity », Int. J. Solids Struct., vol. 44, p. 4978-5002, 2007.

[AVR 08] Avril S., Bonnet M., Bretelle A., Grédiac M., Hild F., Ienny P., LAtourte F., Lemosse D., Pagnacco E., Pierron F., « Overview of identification methods of mechanical parameters based on full-field measurements », Experimental Mechanics, vol. 48, p. 381-402, 2008.

[BEN 95] BEN ABDALLAH J., Inversion gaussienne appliquée à la correction paramétrique de modèles structuraux, Thèse de doctorat, Ecole Polytechnique, Paris, France, 1995.

[BUI 00] Bui H. D., Constantinescu A., «Spatial localization of the error of constitutive law for the identification of defects in elastic bodies », Arch. Mech., vol. 52, p. 511-522, 2000.

[CAL 02] CAlloch S., Dureisseix D., Hild F., «Identification de modèles de comportement de matériaux solides : utilisation d'essais et de calculs », Technologies et Formations, vol. 100, p. 36-41, 2002.

[CHA 99] Chavent G., Kunisch K., Roberts J. E., « Primal-dual formulations for parameter estimation problems. », Raupp M., JR J. D., Koiller J., Menzala G., Eds., Computational and Applied Mathematics, vol. 18, p. 173-229, 1999.

[CON 95] CONSTANTINESCU A., «On the identification of elastic moduli from displacementforce boundary measurements », Inverse Probl. Eng., vol. 1, p. 293-315, 1995.

[DER 01] Deraemaeker A., Sur la maîtrise des modèles en dynamique des structures à partir de résultats d'essais, Thèse de doctorat, ENS Cachan, 2001.

[DER 02] Deraemaeker A., LAdevèze P., LeConte P., «Reduced bases for model updating in structural dynamics based on constitutive relation error. », Comp. Meth. Appl. Mech. Eng., vol. 191, p. 2427-2444, 2002. 
[FEI 06] FeISSEl P., Allix O., « Modified constitutive relation error identification strategy for transient dynamics with corrupted data : the elastic case », Comp. Meth. Appl. Mech. Eng., vol. 196, p. 1968-1983, 2006.

[GER 83] Germain P., Nguyen Q. S., Suquet P., «Continuum thermodynamics », ASME J. Appl. Mech., vol. 50, p. 1010-1020, 1983.

[GER 86] Germain P., Mécanique, Cours de l'Ecole Polytechnique, 1986.

[GEY 02] Geymonat G., Hild F., Pagano S., « Identification of Elastic Parameters by Displacement Field Measurement », C.R. Acad. Sci. Paris, série II, vol. 330, p. 403-408, 2002.

[GEY 03] Geymonat G., PAGAno S., «Identification of mechanical properties by displacement field measurement : a variational approach », Meccanica, vol. 38, p. 535-545, 2003.

[HAD 07a] HADJ-SASSI K., Une stratégie d'estimation conjointe des paramètres et de l'état de structures à comportement non linéaire. Assimilation de données et erreur en loi de comportement, Thèse de doctorat, Ecole Polytechnique, 2007.

[HAD 07b] HADJ-SASSI K., ANDRIEUX S., «Une nouvelle fonctionnelle d'énergie incrémentale totale pour le contrôle des parties réversibles et dissipatives des matériaux standards », Huitième Colloque National en Calcul des Structures, Hermes, p. 255-261, 2007.

[HAL 75] Halphen B., NGuYen Q. S., «Sur les matériaux standards généralisés. », J. Mécanique, vol. 14, p. 39-63, 1975.

[KOH 84] KoHn R., Vogelius M., « Determining conductivity by boundary measurements. », Comm Pure Appl. Math., vol. 37, p. 289-298, 1984.

[KOH 88] KoHn R. V., Lowe B. D., «A variational method for parameter identification », Math. Mod. Num. Anal., vol. 1, p. 119-158, 1988.

[KOH 90] KOHN R., MCKENNEY A., « Numerical implementation of a variational method for electrical impedance tomography. », Inverse Problems, vol. 6, p. 389-414, 1990.

[KOZ 91] Kozlov V. A., MaZ'YA V. G., Fomin A. F., «An iterative method for solving the Cauchy problem for elliptic equations. », Comp. Math. Phys., vol. 31, p. 45-52, 1991.

[LAD 83] LADEVÈze P., LEguillon D., «Error estimate procedure in the finite element method and applications. », SIAM J. Numer. Anal., vol. 20, p. 485-509, 1983.

[LAD 99] LADEVÈze P., ChOUAKI A., «Application of a posteriori error estimation for structural model updating », Inverse Problems, vol. 15, p. 49-58, 1999.

[LAT 07] LATOURTE F., Identification des paramètres d'une loi de Prager et calcul de champs de contraintes dans des matériaux hétérogènes, Thèse de doctorat, Université Montpellier 2, France, 2007.

[LAT 08] Latourte F., Chrysochoos A., Pagano S., Wattrisse B., « Elastoplastic behavior identification for heterogeneous loadings and materials », Exper. Mech., vol. 48, p. 435-449, 2008.

[MOË 99] MoËS N., LAdEvÈze P., DouChIN B., «Constitutive relation error estimators for (visco)plastic finite element analysis with softening », Comp. Meth. Appl. Mech. Eng., vol. 176, p. 247-264, 1999. 
26 Utilisation de la classe ouvrage-hermes

[NGU 08] NGUYen H.-M., Allix O., Feissel P., «A robust identification strategy for ratedependent models in dynamics », Inverse Problems, vol. 24, page065006, 2008.

[REY 90] REYNIER M., Sur le contrôle de modélisations éléments finis : recalage à partir d'essais dynamiques, Thèse de doctorat, Université Pierre et Marie Curie, Paris, France, 1990.

[SIM 98] Simo J. C., Hughes T. J. R., Computational Inelasticity, Springer-Verlag, 1998. 